\title{
Traveling Waves in a Chain of Pulse-Coupled Oscillators
}

\author{
P. C. Bressloff and S. Coombes \\ Nonlinear and Complex Systems Group, Department of Mathematical Sciences, Loughborough University, \\ Loughborough, Leicestershire, LE11 3TU, United Kingdom
}

(Received 11 November 1997)

\begin{abstract}
We derive conditions for the existence of traveling wave solutions in a chain of pulse-coupled integrate-and-fire oscillators with nearest-neighbor interactions and distributed delays. A linear stability analysis of the traveling waves is carried out in terms of perturbations of the firing times of the oscillators. It is shown how traveling waves destabilize when the detuning between oscillators or the strength of the coupling becomes too large. [S0031-9007(98)06205-X]
\end{abstract}

PACS numbers: $87.10 .+\mathrm{e}, 05.45 .+\mathrm{b}$

Many processes in nature can be described in terms of finite chains of coupled nonlinear oscillators. Examples include the undulatory motion of swimming organisms such as the lamprey [1], leech [2], and the Xenopus tadpole [3], peristalsis in vascular and intestinal smooth muscle [4], and synchronization and waves of excitation that arise during sensory processing in the cortex $[5,6]$. Various physical systems such as Josephson junction arrays $[7,8]$ and laser arrays [9] can also be modeled in terms of coupled oscillators. A basic question concerning these systems is the condition under which traveling waves of activity can occur. Traveling waves are typically phase-locked solutions in which each oscillator has the same frequency but the phase varies monotonically along the chain.

Almost all analysis to date has been carried out for chains of oscillators in the weak-coupling regime where averaging methods can be used to reduce the model to a system of phase equations. For a chain of $N+1$ oscillators with nearest-neighbor coupling and natural frequencies $\omega_{n}, n=1, \ldots, N+1$, these take the form [4]

$$
\dot{\theta}_{n}=\omega_{n}+\epsilon \sum_{\langle m, n\rangle} H\left(\theta_{m}-\theta_{n}\right)+O\left(\epsilon^{2}\right),
$$

where $\langle m, n\rangle$ denotes a sum over nearest neighbors of $n$. The periodic interaction function $H$ depends on the nature of the coupling and on the dynamics in a neighborhood of a limit cycle. The parameter $\epsilon$ determines the strength of the interactions with $\epsilon \ll 1$ for weak coupling. Any phase-locked solution has the form $\theta_{n}(t)=\Omega t+\zeta_{n}$, where $\zeta_{n}$ is constant. Substitution of $\dot{\theta}_{n}=\Omega$ into Eq. (1) yields $N$ fixed point equations for the phase differences $\phi_{n}=\theta_{n+1}-\theta_{n}, n=1, \ldots, N$, which are independent of the collective frequency of oscillations $\Omega$; the latter is then determined from the remaining equation $\Omega=\omega_{1}+$ $\epsilon H\left(\phi_{1}\right)$. Note that in general explicitly solving for the fixed points $\left\{\phi_{n}\right\}$ is a nontrivial task since it is necessary to take into account the boundary conditions at the ends of the chain. (In contrast, waves on a circular ring of identical oscillators arise naturally as a consequence of the underlying translational symmetry $[10,11]$.) One finds that there are at least two different mechanisms that can generate traveling wave solutions. The first is based on the presence of a gradient of frequencies along the chain with the wave propagating from the high frequency region to the low frequency region. For example, if $\omega_{n+1}-\omega_{n}=\Delta>0$ in Eq. (1) then traveling waves will occur provided that $\Delta$ is sufficiently small; when the gradient becomes too steep to allow phase locking, two or more pools of oscillators (frequency plateaus) tend to form that oscillate at different frequencies [4]. The second mechanism for wave formation, which can occur in systems of identical oscillators with anisotropic coupling, is due to so-called nondiffusive coupling in which the interaction function $H$ satisfies $H(0) \neq 0$ [12].

In this Letter, we investigate traveling waves in a chain of integrate-and-fire oscillators where the pulselike nature of the interactions between biological oscillators is explicitly incorporated [13]. Pulse-coupled oscillators also arise within the context of certain physical systems such as discrete phase-locked loops [14] and stick-slip models [15]. We derive a set of equations for phase-locked solutions that are structurally identical to those obtained from the phase equation (1), and use this to establish the existence of traveling waves. In contrast to a chain of weakly coupled limit cycle oscillators, our results for integrate-and-fire chains hold for arbitrary values of the coupling $\epsilon$. We also analyze the linear stability of traveling wave solutions for the integrate-and-fire chain and show how the condition for asymptotic stability reduces to that of a corresponding phase model in the weak coupling limit. The existence and stability of traveling wave solutions as a function of the degree of detuning and the strength of coupling between oscillators is investigated through a number of numerical examples. For simplicity, we restrict our discussion to the isotropic case.

Consider a chain of $N+1$ integrate-and-fire oscillators labeled $n=1, \ldots, N+1$. Let $U_{n}(t)$ denote the state of the $n$th oscillator at time $t$. Suppose that the variables $U_{n}(t)$ satisfy the set of coupled equations

$$
\frac{d U_{n}(t)}{d t}=-U_{n}(t)+I_{n}+\epsilon \sum_{\langle m, n\rangle} \hat{E}_{m}(t)
$$

for $0<U_{n}<1$. Equation (2) is supplemented by the condition that the oscillator fires a single pulse or spike 
whenever $U_{n}=1$ and that the state is immediately reset to $U_{n}=0$. In Eq. (2), $I_{n}$ denotes a fixed external input and $E_{n}, n=1, \ldots, N+1$, represents the inputs from the $n$th oscillator. We shall assume that $I_{n}>1$ so that in the absence of any coupling $(\epsilon=0)$ each oscillator fires at a rate of $1 / \bar{T}_{n}$ with $\bar{T}_{n}=\ln \left(I_{n} /\left[I_{n}-1\right]\right)$. The inputs take the form

$$
\hat{E}_{m}(t)=\int_{0}^{\infty} P(\tau) E_{m}(t-\tau) d \tau
$$

for $m=1, \ldots, N+1$, where $E_{m}(t)$ is the sequence of spikes transmitted from the $m$ th oscillator at time $t$ and $P(\tau)$ specifies a distribution of delayed connections. In the particular case of neural systems there are a number of possible sources of delays including axonal transmission delays, synaptic processing, and dendritic processing [11]. Neglecting the shape of an individual pulse, each spike train can be represented as a sequence of Dirac delta functions $E_{n}(t)=\sum_{j=-\infty}^{\infty} \delta\left(t-T_{j}^{n}\right)$ where $T_{j}^{n}$ is the $j$ th firing time of the $n$th oscillator, that is, $U_{n}\left(T_{j}^{n}\right)=1$.

Suppose that we restrict our attention to phase-locked solutions of Eq. (2) in which every oscillator fires with the same fixed period $T$, which has to be determined self-consistently. The state of each oscillator can then be characterized by a constant phase $\theta_{n} \in[0,1]$, and the firing times of the $n$th oscillator become $T_{j}^{n}=(j-$ $\left.\theta_{n}\right) T$. Following Ref. [11], we integrate Eq. (2) over the interval $t \in\left(-T \theta_{n}, T-T \theta_{n}\right)$ using the reset conditions $U_{n}\left(-\theta_{n} T\right)=0$ and $U_{n}\left(T-\theta_{n} T\right)=1$ to obtain the result

$$
\left(1-e^{-T}\right)^{-1}=I_{n}+\epsilon \sum_{\langle m, n\rangle} K_{T}\left(\theta_{m}-\theta_{n}\right)
$$

for $n=1, \ldots, N+1$ where

$$
K_{T}(\theta)=\left[e^{T}-1\right]^{-1} \int_{0}^{T} e^{t} \hat{P}(t+\theta T) d t,
$$

with $\hat{P}(t)=\sum_{j=0}^{\infty} P(t+j T)$ for $0 \leq t<T . \quad \hat{P}(t)$ is extended outside this range by taking it to be a periodic function of $t$ so that $K_{T}(\theta+1)=K_{T}(\theta)$. Any solution of Eq. (4) can be specified in terms of the $N$ phase differences $\Phi=\left(\phi_{1}, \ldots, \phi_{N}\right), \phi_{n}=\theta_{n+1}-\theta_{n}$, and the collective frequency $1 / T$. The phases $\theta_{n}$ are determined only up to an arbitrary uniform phase shift. Comparison of Eq. (4) with the corresponding set of conditions for phase-locked solutions $\left(\dot{\theta}_{n}=\Omega\right.$ ) of Eq. (1) shows that they are formally identical, with $K_{T}$ playing the role of the phase interaction function $H$ and $\omega_{n} \rightarrow I_{n}, \Omega \rightarrow$ $\left(1-e^{-T}\right)^{-1}$. It follows that various methods previously developed to establish the existence of traveling waves in chains of weakly coupled limit cycle oscillators can be carried over to the case of integrate-and-fire chains. Before illustrating this, however, a number of important differences between phase-locked solutions of Eqs. (1) and (4) need to be highlighted. First, Eq. (4) is exact, whereas Eq. (1) is valid only to $O(\epsilon)$ since it is derived under the assumption of weak coupling. Thus in the former case, analysis of phase locking can be continued to the strong coupling regime. Second, unlike $H(\theta)$, $K_{T}(\theta)$ explicitly depends on the collective frequency of oscillations $1 / T$ and the latter must be determined self-consistently.

The third significant difference is that integrate-andfire oscillators are of type I, which means that an instantaneous excitatory stimulus always advances its phase, whereas limit cycle oscillators are typically of type II since such a stimulus can either advance or retard the phase, depending upon the point on the cycle at which the stimulus is applied [16]. This distinction manifests itself in the nature of the coupling functions $H(\theta)$ and $K_{T}(\theta)$. In the case of purely excitatory interactions, only the former takes on both positive and negative values over the domain $\theta \in[0,1]$, whereas $K_{T}(\theta)$ is a positive function. In order to allow for more general forms of the interaction function $K_{T}(\theta)$, we shall assume in Eq. (2) that there exists a combination of excitatory $(+)$ and inhibitory $(-)$ interactions between the integrate-and-fire oscillators, such that $P(\tau)=P^{+}(\tau)-P^{-}(\tau)$ with $P^{ \pm}(\tau) \geq 0$. Such combinations are found, for example, in neural circuits underlying locomotion [3] and cortical microcircuits [17], where there is a mixture of long and short range excitation and short range inhibition mediated through synaptic interactions. In fact both inhibitory and excitatory post synaptic potentials (PSPs) have some finite rise and fall time. Moreover, the former are often generated by inhibitory interneurons leading to another form of delay in the interaction process. For simplicity, we consider a situation with nearest-neighbor interactions in which the inhibitory pathway is delayed with respect to the excitatory one and describe both forms of PSP with a so-called $\alpha$ function $g(\tau)=\alpha^{2} \tau e^{-\alpha \tau}$, where $\alpha$ is the inverse rise time. The interactions may be written as $P^{+}(\tau)=g(\tau)$ and $P^{-}(\tau)=g\left(\tau-\tau_{d}\right) \Theta\left(\tau-\tau_{d}\right)$, where $\tau_{d}$ is the delay associated with the inhibitory pathway and $\Theta(x)=1$ if $x \geq 0$ and is zero otherwise.

If the number of oscillators is sufficiently large and the frequency gradient is sufficiently small, then phaselocked solutions of Eq. (4) can be analyzed by extending a continuum approximation method developed by Kopell and Ermentrout for weakly coupled limit cycle oscillators [12]. We shall briefly indicate the underlying idea and present details elsewhere [18]. First, let $f_{T}$ and $g_{T}$ be the even and odd parts of $K_{T}$, respectively, and define $\Omega=\left(1-e^{-T}\right)^{-1}$. Rewrite Eq. (4) in terms of $f_{T}, g_{T}$ and then consider the following continuum approximation (for $N$ large but finite)

$$
\Omega=I(x)+\epsilon\left[2 f_{T}(\phi)+\frac{1}{N}\left[g_{T}(\phi)\right]_{x}\right]
$$

supplemented by the boundary conditions $\Omega=I(0)+$ $\epsilon\left[f_{T}(\phi)+g_{T}(\phi)\right] \quad$ at $\quad x=0 \quad$ and $\quad \Omega=I(1)+$ $\epsilon\left[f_{T}(\phi)-g_{T}(\phi)\right]$ at $x=1$. Here $I(x)$ is a smooth 
function of $x, 0 \leq x \leq 1$, such that $I_{n}=I(n /[N+1])$ and $\phi(x)$ is a smooth approximation of $\phi_{n}$, that is, $\phi_{n} \approx \phi(n /[N+1])$. The next step is to assume that $T$ is fixed, so that $f_{T}$ and $g_{T}$ are known functions, and solve Eq. (6) for large $N$. This generates a singularly perturbed two-point boundary value problem for $\phi(x)$, which can be solved using the method of matched asymptotic expansions $[12,18]$. The latter involves piecing together an outer solution, which has sufficiently slow spatial variation so that the term $N^{-1}\left[g_{T}(\phi)\right]_{x}$ can be neglected in Eq. (6), and an inner solution describing a rapidly varying boundary layer. Imposing certain conditions on the functions $f_{T}$ and $g_{T}$, this yields a unique solution to the given boundary value problem including a value for $\Omega$, which we denote by $\Omega(T)$. If $T$ is now treated as a free variable we obtain a one-parameter family of solutions with $\Omega(T)$ a known function of $T$. A unique, self-consistent, phase-locked solution to Eq. (6) then exists provided that there exists a unique period $T$ satisfying $\Omega(T)=\left(1-e^{-T}\right)^{-1}$, and such that the original hypotheses on the coupling functions $f_{T}$ and $g_{T}$ are not violated [18]. Finally, it can be proven that the original discrete model of Eq. (4) has a solution that converges to the solution of the continuum model (nonuniformly in the boundary layer) in the limit $N \rightarrow \infty$. An example of a boundary layer is shown in Fig. 1, where a traveling wave solution obtained by numerically solving Eq. (4) is compared with the solution of the continuum model given by Eq. (6).

The linear stability of a phase-locked solution $(\Phi, T)$ of Eq. (2) can be determined along similar lines to the analysis of globally coupled integrate-and-fire oscillators [19] by considering small perturbations of the phase-locked firing patterns $T_{j}^{n}=\left(j-\theta_{n}\right) T+\delta_{j}^{n}$. Solving Eq. (2) and the reset condition leads to an implicit map for the per-

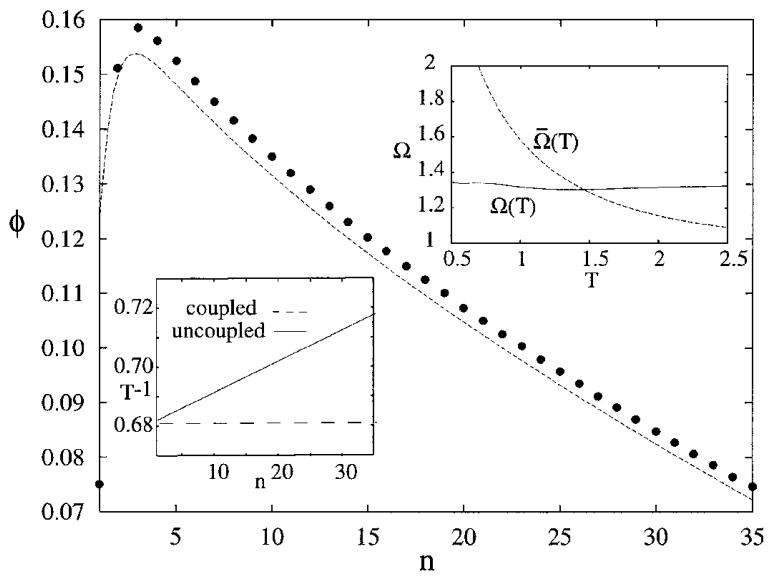

FIG. 1. A traveling wave solution for $N=36, \tau_{d}=0.6$, $\alpha=10, \epsilon=0.1, \beta=0.01 \epsilon$, and $I_{1}=1.3$. The points represent the numerical solution of Eq. (4), while the solid line is the solution of the corresponding continuum boundary value problem. The right inset shows how the intersection of $\Omega(T)$ and $\bar{\Omega}(T)=\left[1-e^{-T}\right]^{-1}$ yields a unique value for the collective period, $T=1.47$. turbations $\delta_{j}^{n}$. Linearizing this map yields a linear delaydifference equation that has solutions of the form $\delta_{k}^{n}=$ $\lambda^{k} \delta_{n}$ with the eigenvalue $\lambda$ satisfying the equation [18]:

$$
\begin{aligned}
(\lambda-1) A_{n}^{T}(\Phi) \delta_{n}= & \sum_{j=0}^{\infty} \sum_{\langle m, n\rangle} a_{n m, j}^{T}(\Phi) \\
& \times\left[\lambda^{-j} G_{n m}(\lambda) \delta_{m}-\delta_{n}\right],
\end{aligned}
$$

where $G_{n m}(\lambda)=\lambda$ if $\theta_{n}<\theta_{m}$ and $G_{n m}(\lambda)=1$ if $\theta_{n}>$ $\theta_{m}$ and

$$
\begin{aligned}
A_{n}^{T}(\Phi) & =\left[I_{n}-1+\epsilon \sum_{\langle m, n\rangle} \hat{P}\left(\left(\theta_{m}-\theta_{n}\right) T\right)\right], \\
a_{n m, j}^{T}(\Phi) & =\epsilon e^{-T} \int_{0}^{T} e^{t} P^{\prime}\left(t+\left(j+\theta_{m}-\theta_{n}\right) T\right) d t .
\end{aligned}
$$

Here / indicates differentiation with respect to $t$. Note that one solution to Eq. (7) is given by the eigenvalue $\lambda_{0}=1$ and its corresponding eigenvector $\delta_{m}=\delta$ for all $\mathrm{m}$. This reflects the invariance of the dynamics with respect to a uniform shift in the firing times of the oscillators. The condition for asymptotic stability of a traveling wave solution is $|\lambda|<1$ for all eigenvalues $\lambda \neq \lambda_{0}$. In the weak-coupling limit, with $I_{n}=I$ for some fixed $I>1$, solutions to Eq. (7) in the complex $\lambda$ plane will either be in the neighborhood of the real solution $\lambda=1$ or in the neighborhood of one of the poles of $\sum_{j=0}^{\infty} a_{n m, j}^{T}(\Phi) \lambda^{-j} G_{n m}(\lambda)$. These poles all lie inside the unit circle and hence are not important in terms of determining whether or not a phase-locked solution is stable. Thus to first order in $\epsilon$ we set $\lambda=1$ and $T \rightarrow \bar{T}=\ln (I /[I-1])$ on the right-hand side of (7). The result is that to $O(\epsilon)$ the spectrum close to $\lambda=1$ coalesces into $N+1$ distinct points given by $\lambda_{0}=1$ and $\lambda_{p}=1+\chi \mu_{p}$, $p=1, \ldots, N$, where $\chi=\epsilon\left[1-e^{-\bar{T}}\right] /(\bar{T}[I-1])$ and $0, \mu_{1}, \ldots, \mu_{p}$ are the eigenvalues of the $N+1 \times N+1$ matrix $\hat{J}_{m n}(\Phi)=J_{m n}(\Phi)-\delta_{m, n} \sum_{\langle p, n\rangle} J_{p n}(\Phi) \quad$ with $J_{m n}(\Phi)=\sum_{\langle p, n\rangle} K_{\bar{T}}^{\prime}\left(\theta_{p}-\theta_{n}\right) \delta_{m, p}$. The condition for stability reduces to $\operatorname{Re} \mu_{p}<0$ for all $p=1, \ldots, N$. An identical stability condition would be found for phaselocked solutions of Eq. (1) with $H \rightarrow K_{\bar{T}}$. This can be understood by noting that in the weak coupling limit an averaging procedure applied to Eq. (2) leads to such a phase model [11].

We deduce from the above linear stability analysis that in the weak coupling limit the behavior of the integrateand-fire chain will be similar to that found previously for the phase-coupled model (1). However, we expect new features to emerge as the coupling is increased. To investigate this further it is useful rewrite Eq. (4) in terms of the $N$ phase differences $\phi_{n}$ assuming a constant input gradient $I_{n+1}-I_{n}=\beta$ :

$$
\begin{aligned}
0=\beta+\epsilon[ & K_{T}\left(\phi_{n+1}\right)-K_{T}\left(\phi_{n}\right)+K_{T}\left(-\phi_{n}\right) \\
& \left.-K_{T}\left(-\phi_{n-1}\right)\right],
\end{aligned}
$$




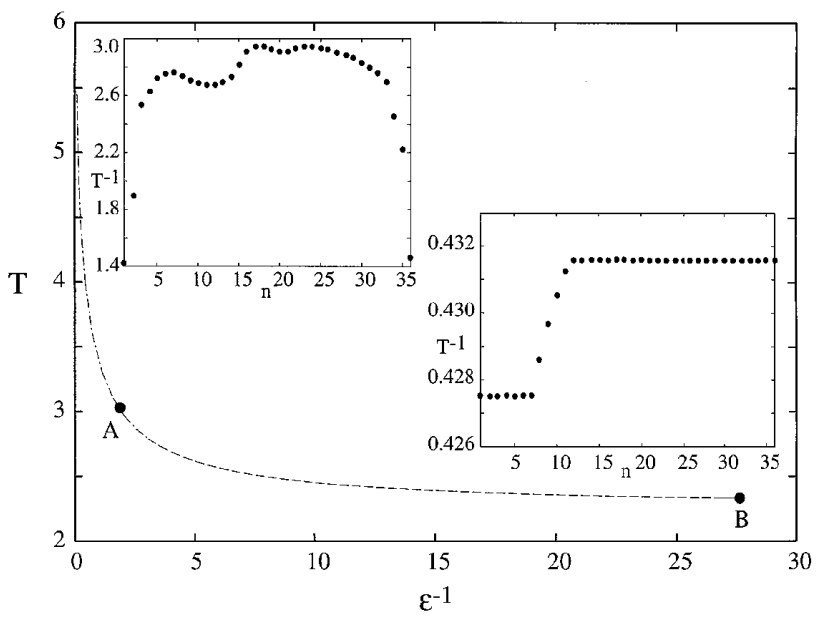

FIG. 2. Numerical continuation of a phase-locked solution into the strong coupling regime with $N=36, \tau_{d}=1.0, \alpha=$ $8, \beta=0.0005$, and $I_{1}=1.1$. Such solutions may destabilize if one or more of the eigenvalues of the linearized firing map cross the unit circle and new stable solutions may appear. Numerical support for this possibility is provided in the left inset where we show a stable nonfrequency-locked solution which exists at the point $A(\epsilon=0.4)$ in the strong coupling regime. At point $B(\epsilon=0.03)$, frequency locked states cease to exist in favor of a stable two-plateau state in which each group of oscillators has a differing frequency, as shown in the right inset.

$$
\left(1-e^{-T}\right)^{-1}=I_{1}+\epsilon K_{T}\left(\phi_{1}\right),
$$

and the boundary conditions are taken to be $K_{T}\left(-\phi_{0}\right) \equiv$ $0 \equiv K_{T}\left(\phi_{N+1}\right)$. The parameter $\beta$ determines the amount of detuning of the oscillator frequencies. In the weak coupling limit with $T \rightarrow \bar{T}$, phase-locked solutions will depend only on the parameters $\epsilon, \beta$ through their ratio $\beta / \epsilon$. As previously found for weakly coupled phase oscillators [4], we would expect that increasing the level of detuning $\beta$ for fixed $\epsilon$ (small) leads to the disappearance of stable phase-locked solutions and the formation of frequency plateaus. This is indeed the case as shown in the right-hand inset of Fig. 2, which was obtained by direct integration of Eq. (2). Outside the weak coupling regime, on the other hand, Eqs. (10) and (11) cannot be solved for the phase differences $\phi_{n}$ independently of the collective period $T$. The latter is determined selfconsistently to yield $T=T(\epsilon, \beta)$. Thus, phase-locked solutions now depend on both parameters $\epsilon, \beta$ independently. If $\epsilon$ is increased for fixed $\beta$ it is possible for phase-locked solutions to persist into the strong coupling regime (see Fig. 2). However, increasing the coupling strength $\epsilon$ can induce instabilities due to one or more eigenvalues of Eq. (7) crossing the unit circle leading to new forms of solution in which the bulk of the oscillators fire at approximately twice the frequency of the boundary ones (see left-hand inset of Fig. 2). Note that in contrast to the weak-coupling regime, destabilization of a phaselocked solution no longer requires that eigenvalues cross the unit circle close to $\lambda=1$. Indeed, preliminary studies suggest that the mechanism of destabilization is very distinct from that occurring for small $\epsilon$ and large $\beta$. We hope to pursue this issue further elsewhere.

We conclude that a gradient of synaptic drive can sustain a stable traveling wave, with realistic neuronal firing frequencies, for a large range of coupling strengths. Importantly, such a mechanism is believed to underly the swimming behavior observed in Xenopus embryos, where a rostral-caudal gradient in synaptic drive is believed to be sustained by positive feedback within the premotor neural circuitry [3]. A more detailed model based on the work in this Letter is being used to test such hypotheses.

This research was supported by Grant No. GR/K86220 from the EPSRC (U.K.).

[1] A. H. Cohen, P. J. Holmes, and R. H. Rand, J. Math. Biol. 13, 345-369 (1982).

[2] W. O. Friesen and R. A. Pearce, Semin. Neurosci. 5, 41 (1993).

[3] A. Roberts, Sci. Prog. (Oxford) 74, 31 (1990).

[4] G. B. Ermentrout and N. Kopell, SIAM J. Math Anal. 15, 215 (1984).

[5] M. Wilson and J.M. Bower, J. Neurophysiol. 67, 981995 (1992).

[6] C. M. Gray and W. Singer, Proc. Natl. Acad. Sci. U.S.A. 86, 1698 (1989).

[7] P. Hadley, M. R. Beasley, and K. Wiesenfeld, Phys. Rev. B 38, 8712 (1988).

[8] S. Watanabe and S.H. Strogatz, Physica (Amsterdam) 74D, 197 (1994).

[9] M. Silber, L. Fabiny, and K. Wiesenfeld, J. Opt. Soc. Am. B 10, 1121 (1993).

[10] G. B. Ermentrout, J. Math. Biol. 23, 55 (1985).

[11] P. C Bressloff, S. Coombes, and B. de Souza, Phys. Rev. Lett. 79, 2791-2794 (1997).

[12] N. Kopell and G. B. Ermentrout, SIAM J. Appl. Math. 50, 1014 (1990).

[13] R. E Mirollo and S. H. Strogatz, SIAM J. Appl. Math. 50, 1645 (1990).

[14] G. Goldsztein and S. H. Strogatz, Int. J. Bifurcation Chaos Appl. Sci. Eng. 5, 983 (1994).

[15] S. Bottani, Phys. Rev. Lett. 74, 4189 (1995).

[16] D. Hansel, G. Mato, and C. Meunier, Europhys. Lett. 23, 367 (1993).

[17] G. M. Shepherd, The Synaptic Organization of the Brain (Oxford University Press, New York, 1990).

[18] P. C Bressloff and S. Coombes (unpublished).

[19] C. van Vreeswijk, Phys. Rev. E 54, 5522 (1996). 